\title{
OPEN Study the plasmonic property of gold nanorods highly above damage threshold via single-pulse spectral hole-burning experiments
}

\author{
Zibo Wang ${ }^{\bowtie}$, Zhe Kan \& Mengyan Shen
}

Intense femtosecond laser irradiation reshapes gold nanorods, resulting in a persistent hole in the optical absorption spectrum of the nanorods at the wavelength of the laser. Single-pulse hole-burning experiments were performed in a mixture of nanorods with a broad absorption around $800 \mathrm{~nm}$ with a 35 -fs laser with $800 \mathrm{~nm}$ wavelength and $6 \mathrm{~mJ} /$ pulse. A significant increase in hole burning width at an average fluence of $10^{6} \mathrm{~J} / \mathrm{m}^{2}$ has been found, suggesting a tripled damping coefficient of plasmon. This shows that the surface plasmonic effect still occurs at extremely high femtosecond laser fluences just before the nanorods are damaged and the remaining $10 \%$ plasmonic enhancement of light is at the fluence of $10^{6} \mathrm{~J} / \mathrm{m}^{2}$, which is several orders of magnitude higher than the damage threshold of the gold nanorods. Plasmon-photon interactions may also cause an increase in the damping coefficient.

Gold nanorods attract our attention due to their applications in medicine, chemistry and physics, including biosensing $^{1}$, drug delivery ${ }^{2}$, cellular imaging ${ }^{3,4}$, cancer therapy ${ }^{5}$, chemical analysis ${ }^{6,7}$, catalysts ${ }^{8-12}$, electronics ${ }^{13,14}$ and nonlinear processes ${ }^{15}$. These applications utilize the plasmonic effect: the interaction of conductive electrons in the metallic structures with electromagnetic fields ${ }^{16}$. The effects of the particles' size, shape, and environment on plasmonic absorption have also been studied extensively ${ }^{17,18}$. In particular, gold nanoparticles are especially favored for their physical and chemical stability as well as the tunability of their plasmonic properties ${ }^{19}$.

The resonant plasmons lead to a nano-focusing effect, or electromagnetic (EM) enhancement, by coupling a resonating bulk optical radiation into the strongly localized near field ${ }^{20,21}$. The simplest expression that describes the EM enhancement for a small spherical particle can be written as:

$$
\frac{E_{\text {local }}}{E_{0}} \propto \frac{1}{\varepsilon(\omega)+2 \varepsilon_{s}}
$$

where $E_{\text {local }}$ is the local electric field due to the polarization of the particle, $\omega$ is the frequency of the incident light, $\varepsilon(\omega)$ and $\varepsilon_{\mathrm{s}}$ are the dielectric constants of the small metal including that of its surrounding at the frequency $\omega$, and $E_{0}$ is the electric field of the incident light. According to this equation, the local electric field will approach infinity when the dielectric constant approaches $-2 \varepsilon_{s}$, however the imaginary part of the dielectric constant keeps the EM enhancement finite. The light intensity enhancement factor $\left(\frac{E_{\text {local }}}{E_{0}}\right)^{2}$ is approximately proportional to $\frac{1}{\Gamma^{2}}$, where $\Gamma$ is the damping coefficient to describe the electron scattering in the Drude model. The light intensity enhancement factor reaches the order of $10^{422}$ which is due to weak light-nanoparticle interactions. Whether this enhancement factor is still large when the laser energy is high, can be investigated by studying the light intensity dependence of the homogeneous broadening of $\Gamma$, which can be directly obtained from the measurement of the confocal light scattering spectroscopic microscopy ${ }^{23}$ and a scanning near-field optical microscopy of single particles ${ }^{24}$. $\Gamma$ can also be obtained from the optical absorption and the size/shape distributions of an assembly of particles ${ }^{24}$. However, these methods do not provide instantaneous information of $\Gamma$ right before the time that the nanoparticles are melted or damaged when they interact with an intense femtosecond laser pulse.

The homogeneous broadening of $\Gamma$ of a gold nanoparticle is several tens of $n m$, which is much larger than that of semiconductor nanoparticles. When light irradiates a semiconductor nanoparticle, the particle may be 


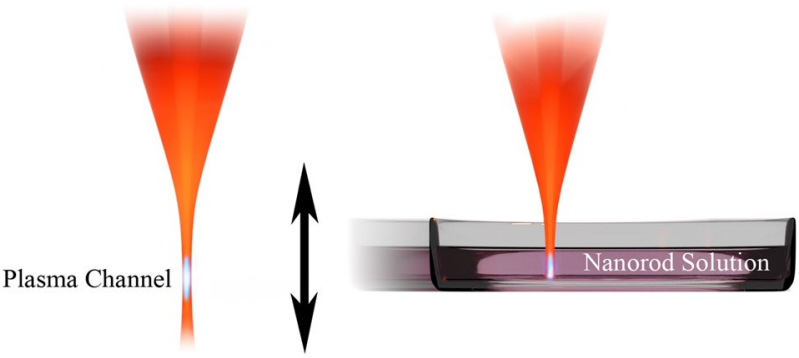

Figure 1. An illustration of the experimental setup for the femtosecond laser irradiation, where the laser is focused vertically as shown in orange. The drawing on the left shows the plasma channel (in bright blue) formed in the air and the drawing on the right shows the surface of the nanorod solution is placed above the air plasma channel position. The cuvette can move vertically with a translation stage to vary the laser spot size on the surface of the solution.

photoionized ${ }^{25}$, which results in a photodarkening effect ${ }^{26}$, spectral diffusion $^{27,28}$, and a persistent hole-burning phenomenon ${ }^{29}$. The hole-burning effect provides an effective method to study the homogeneous broadening of the optical spectrum of a semiconductor nanoparticle ${ }^{29}$.

Kudryashov et al. reported a broadening of the localized plasmonic resonance at about $520 \mathrm{~nm}$ of gold spheres that are $160-\mathrm{nm}$ in diameter when irradiated by a $100-\mathrm{fs}$ pulse laser at $800 \mathrm{~nm}$ with an average fluence of about $10^{4} \mathrm{~J} / \mathrm{m}^{230}$. This phenomenon was attributed to an increase in the free carrier concentration in s-band, which is induced by strong excitation and electron heating ${ }^{30}$. Here, we report the study of resonantly exciting the longitudinal plasmon of gold nanorods at $800 \mathrm{~nm}$. When an intense laser pulse irradiates Au nanorods, the nanorods absorb the light through the resonant-plasmon excitation or weak non-resonant excitation. The absorbed energy heats up the nanorods, and the rods may be deformed or damaged ${ }^{31}$. This may result in a persistent hole in the absorption spectra. The hole width is due to the homogeneous broadening $\Gamma$ of the resonant plasmon excitation because the non-resonant excitation cannot generate a hole but a broader spectrum shift. The inhomogeneous absorption band of an assembly of gold nanoparticles is generally a little broader than homogeneous broadening nanoparticles ${ }^{24}$. We have mixed gold nanorods with different sizes, similarly to DeSantis et al. ${ }^{32}$ to increase the inhomogeneous absorption band, but we used a single-pulse design to eradicate influences from previous laser pulse irradiations. The damping factor of nanorods can thus be studied explicitly under a simplified process of the single pulse-irradiation. Here, we report a laser intensity dependence of the $\Gamma$ obtained with this single pulse hole burning method.

A significant increase in hole burning width from 80 to $250 \mathrm{~nm}$ at $10^{6} \mathrm{~J} / \mathrm{m}^{2}$ fluences has been found, suggesting a tripled damping coefficient of plasmon. This fluence is $10^{4}$ times higher than the threshold for gold nanorods to be completely damaged, which is experimentally estimated to be $100 \mathrm{~J} / \mathrm{m}^{2}$ with femtosecond pulses ${ }^{33}$. The increase in hole burning width shows that the surface plasmonic effect holds at extreme femtosecond laser fluences, achieving $10 \%$ plasmonic enhancement of light at the fluence of about $10^{6} \mathrm{~J} / \mathrm{m}^{2}$. The results suggest that an appropriate gold nanostructure can still be used to enhance or nano-focus an intensive femtosecond laser pulse. For example, potential applications are for triggering nuclear reactions ${ }^{34}$ and for generating coherent high-energy particle beams. By using a plasmon-photon interaction ${ }^{35}$, we understand the increase of the damping coefficient because the boson property of photons cannot be neglected when the photon number or light intensity is high.

\section{Experimental}

We used gold nanorods made by Nanopartz INC which are $10 \mathrm{~nm}$ in diameter and their length ranges from 20 to $50 \mathrm{~nm}$. The nanorods are dissolved in water and citrate is used as the ligand. The concentration is $2 \times 10^{12}$ rod per $\mathrm{ml}$ before dilution. A femtosecond laser of $800 \mathrm{~nm}$ in wavelength, repetition rate of $1 \mathrm{kHz}$, pulse duration of $35 \mathrm{fs}$, and pulse energy of $6 \mathrm{~mJ}$ were used to excite the gold nanorods. To prevent the 35 -fs laser pulse from stretching, the horizontal laser beam was directly tightly focused $200 \mathrm{~mm}$ below the beam with an off-axis parabolic goldcoated mirror. The focal point of the mirror was placed below the top surface of the sample nanorod solution with a depth of about $3 \mathrm{~mm}$. A translation stage carrying the solution in a glass petri dish is moved vertically to adjust the spot size upon contact, varying the fluence from 300 to $10^{6} \mathrm{~J} / \mathrm{m}^{2}$ as shown in Fig. 1. A variable neutraldensity filter was also used to vary the energy of each pulse so the fluence ranges from 10 to $300 \mathrm{~J} / \mathrm{m}^{2}$. An air plasma channel (or filament) will form around the focal point if the sample is removed as illustrated in Fig. 1. The length of the air plasma channel was observed to be about $2.5 \mathrm{~mm}$. For the maximum fluence excitation, the focal point of the mirror was placed about $2 \mathrm{~mm}$ below the front surface of the sample nanorod solution, so that the laser fluence is lower than the plasma channel formation threshold in the air before the light enters the nanoparticle solution ${ }^{36}$ as shown in Fig. 1 . The spot size of the laser just before the light entering the solution is estimated to be about $50 \mu \mathrm{m}$ (which is also the size of the cross-section of the following filament in the solution) for calculating the maximum fluence. The minimum spot size of laser damage on a silicon surface in water is measured to be about $50 \mu \mathrm{m}$, which agrees with the estimation ${ }^{37-39}$.

After the light enters the solution, its energy is not uniformly distributed in the plasma channel because of the self-focusing and filament formation. The average separation between nanorods in the solution is estimated 


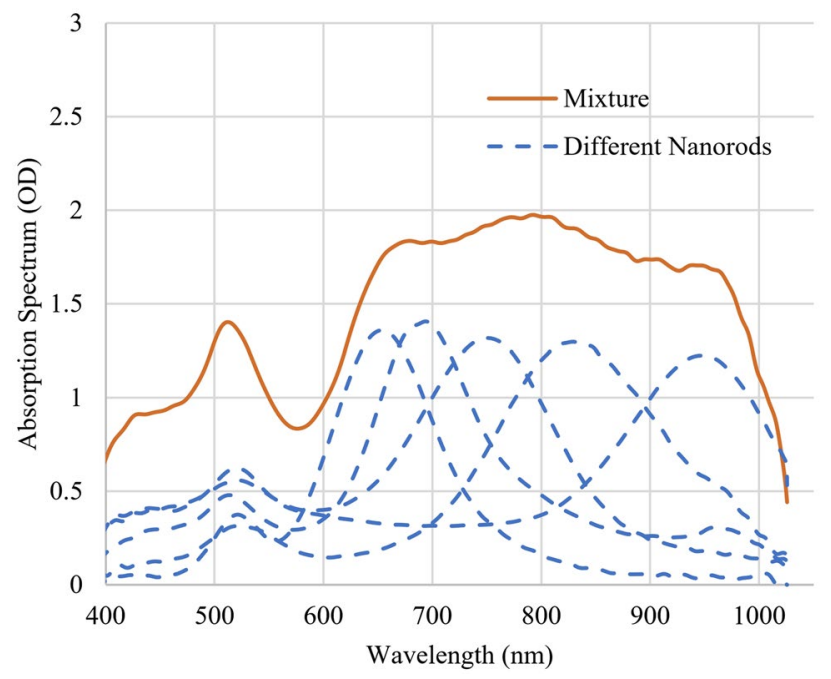

Figure 2. Absorption spectra of different nanorods. The dashed spectra with absorption peaks at 650, 700, 750, 808 , and $950 \mathrm{~nm}$ are of gold nanorods with the average aspect ratios of 2.4, 2.9, 3.5, 4.1, and 5.5, respectively. The solid line represents the absorption spectrum of the mixture of the five different nanorods with the average aspect ratios from 2.4 to 5.5 .

to be $3 \mu \mathrm{m}$, the size of the optical cross-section of a nanorod is more than a hundred of nanometers which is several times larger than its geometry size ${ }^{40,41}$. The laser energy delivered into the nanorod may be much larger than the direct estimation with the laser fluence and its geometry size, which needs further study. Therefore, the fluence obtained with the laser pulse energy and the laser spot size is an average fluence. i.e., the fluences for the following experiments are of average fluences.

When the laser propagates in the solution for a distance of about $3 \mathrm{~mm}$, white light is generated ${ }^{30}$. The white light with a broad spectrum may uniformly excite and deform all the nanorods with different sizes ${ }^{19}$, which cannot generate a hole but a broad spectrum shift or change. Thus, the white light does not contribute to the width of the spectral hole formed with the resonant excitation at $800 \mathrm{~nm}$ in this work.

During the experiment, an optical chopper wheel chopped the pulse train into $50 \mathrm{~Hz}$ and another electric shutter further reduced the repetition rate to $2 \mathrm{~Hz}$. Irradiation time was actively controlled by a programmable shutter, and a translation stage moves the sample at about $1 \mathrm{~mm} / \mathrm{s}$ horizontally as shown in Fig. 1 . This setup gives enough time for the sample to move to a new position for single pulse irradiation, since nanoparticles move slowly in a liquid ${ }^{42}$. Single-pulse irradiation scanning prevents most of the nanorods from being damaged by multiple pulses and decreases the chance of the nanorods from being reshaped or damaged from further irradiation. This scanning technique makes the measurement possible for the homogeneous broadening of a nanorod's plasmon resonance.

The absorption spectra of the sample solutions in a cuvette with 1-cm length optical path were measured using an OceanView Vis-NIR spectrometer and a tungsten light source. To investigate the homogeneous broadening of a nanorod's plasmon resonance with the hole burning method, solutions of nanorods with an aspect ratio ranging from 2.4 to 5.5 were used. Resonance peaks ranging from 650 to $950 \mathrm{~nm}$ (dashed lines in Fig. 2) were chosen and mixed to create a spectral plateau from 650 to $950 \mathrm{~nm}$ as shown in the solid line in Fig. 2. An optical density (OD) equal to 1 corresponds to $10 \%$ transmitted light through the samples. This mixture was used to capture interactions of lasers with nanoparticles that have different resonance wavelengths. Non-resonance excitations ${ }^{43}$ do not generate hole structures because nanorods of different sizes and shapes respond uniformly to the light while the resonance plasmon excitation generates a hole in the spectrum ${ }^{32}$. As shown in Fig. 2, the absorption spectra in dashed lines are a wide range of nanorods with different nanorods and the absorption spectrum of their mixture is shown in solid line. A plateau of absorption is obtained in the spectrum of the mixture.

The mixture of gold nanorods with different aspect ratios was placed on a polished silicon wafer and dried in hot air at $50{ }^{\circ} \mathrm{C}$. With a field emission scanning electron microscope (SEM) (JEOL JSM-7401F), SEM images were taken as shown in Fig. 3. The sizes of gold particles range from $10 \mathrm{~nm} \times 70 \mathrm{~nm}$ to $15 \mathrm{~nm} \times 25 \mathrm{~nm}$ with an aspect ratio of 1.6 to 7 , and correspond to longitudinal resonance wavelengths of $550-1000 \mathrm{~nm}$.

By comparing SEM images of gold nanorods before and after single pulse scanning, nano spheres with radii ranging from 10 to $30 \mathrm{~nm}$ could be found. This result is in agreement with the deformation results proposed by DeSantis et al. ${ }^{32}$. Nanorods with longer aspect ratio $(>6.75)$, could still be seen, while intermediate aspect ratios (3-5) have reduced in portions. The aspect ratio was obtained by measuring the long and short axes of the nanorods in SEM images, and a histogram of its percentage frequency is shown in Fig. 4.

This result coincides with the transmission spectrum that the absorption peak is burned around $800 \mathrm{~nm}$, which corresponds to resonance frequency of gold nanorods of an aspect ratio of 4.1. The formation of nanospheres from the damage of nanorods loses the original longitudinal resonance wavelength around $800 \mathrm{~nm}$, leaving an increased transverse wavelength at $520 \mathrm{~nm}$. The persistence of long aspect ratio nanorods also supports 

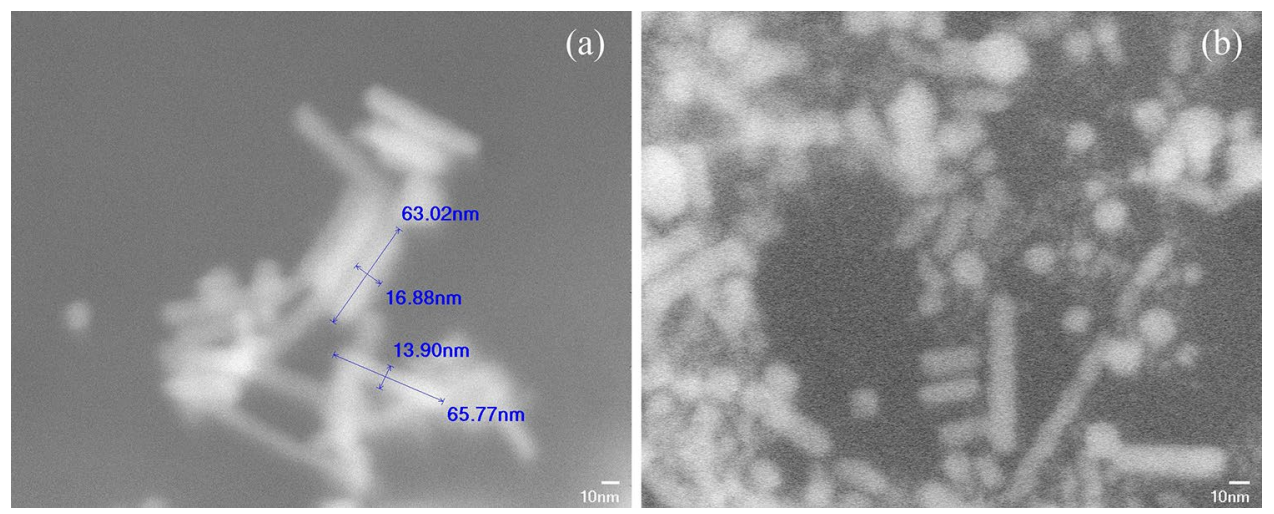

Figure 3. SEM image of an assembly of nanorods. (a) before irradiation, nanorods with different sizes and aspect ratio can be seen with length from 30 to $70 \mathrm{~nm}$ and width ranging from 10 to $18 \mathrm{~nm}$. (b) Nanorods after being irradiated at $10^{6} \mathrm{~J} / \mathrm{m}^{2}$.

that persistent hole burning method can be used for the study of the homogeneous broadening $\Gamma$ of the resonant plasmon excitation up to the fluence at $10^{6} \mathrm{~J} / \mathrm{m}^{2}$.

\section{Results and analysis}

The absorption spectra of nanorod solutions before and after laser irradiation was taken and an example in Fig. 5 presents the spectra for femtosecond laser fluence at $300 \mathrm{~J} / \mathrm{m}^{2}$. A distinctive decrease of the optical absorption around $800 \mathrm{~nm}$ can be observed, which is much wider than the spectral width of laser as shown in dashed line.

The spectra were denoised using a Fourier method by filtering out high frequency components. Then the spectra before the irradiation was divided by that after the irradiation to get a ratio spectrum. A decrease in absorption spectrum will result in an increase in the ratio. Typical results are shown in Fig. 6 . An obvious peak at about $800 \mathrm{~nm}$ could be seen, which is the wavelength of the laser, indicating that the nanorods that resonate with the laser wavelength are destroyed the most. This is very different from the result of DeSantis et al. ${ }^{32}$. The hole peak wavelength was very different from the laser wavelength because their nanorods were excited by multiple laser pulses and after a few pulses, the nanorods had already been deformed. A decrease at $\sim 520 \mathrm{~nm}$ could be seen at higher intensities, corresponding to transverse plasmonic modes of nanospheres and nanorods. It suggests an increase in transverse modes of surface plasmon, which could be found in both nanorods and nanospheres. This result supports the theory of laser induced deformation: nanorods with the 800 -nm longitudinal resonance wavelength were melted into spheres with only $520 \mathrm{~nm}$ transverse modes.

Figure 6 shows the ratio of absorption spectrum before irradiation to that of after irradiation with laser at various fluences. (a) Shows the ratio of spectra before and after being irradiated at a fluence of $220 \mathrm{~J} / \mathrm{m}^{2}$. An ablation of spectrum at $800 \mathrm{~nm}$ can be seen. (b) Shows the ratio of spectra laser with a fluence of $1.1 \times 10^{4} \mathrm{~J} /$ $\mathrm{m}^{2}$. There is the change at $800 \mathrm{~nm}$, and the generation of nanospheres with transverse resonance wavelength of $520 \mathrm{~nm}$ results in a dip. (c) Shows the ratio of spectra before and after laser irradiation at a fluence of $2.46 \times 10^{6} \mathrm{~J} /$ $\mathrm{m}^{2}$. The hole burning effect took place rapidly and peaked at about $800 \mathrm{~nm}$. The hole burning width is measured at the full width at half maximum. If no clear baseline is available, as shown in Fig. 6b,c 1 is used as baseline to calculate half maximum and the resulting widths are shown with dashed lines. Since a femtosecond laser adds extra width from its spectrum, this extra width was canceled by comparing the final results with a convolution of a gaussian distribution and the spectrum of the laser, which lead to a $\sim 3 \mathrm{~nm}$ decrease. Other broadening effects, including the inter-band transitions ${ }^{44,45}$, temperature ${ }^{46}$ and size ${ }^{47}$ broadening, could be eradicated using a similar convolutional fitting process. These broadening contributions are comparatively small as the spectral hole increases to more than $200 \mathrm{~nm}$, and thus are neglected. The width of the spectral hole increases as intensity increases, implying an increased damping coefficient (i.e., the plasmonic enhancement decreases). Since the number of pulses to create a significant spectral hole varies for different fluences, the experimental conditions varies and thus the absolute ratio is not identical.

The results were plotted as a function of laser fluence as shown in Fig. 7. The hole width slowly increased from $149 \mathrm{~nm}$ at $11 \mathrm{~J} / \mathrm{m}^{2}$ to $248 \mathrm{~nm}$ at $3 \times 10^{6} \mathrm{~J} / \mathrm{m}^{2}$, compared with low-fluence limit $80 \mathrm{~nm}$, which is labeled by the dashed line. Qiu et al. ${ }^{24}$ predicted that when the wavelength of the plasmon resonance of a nanorod increases, its homogeneous broadening also increases. They used a scanning near-field optical microscopy to obtain a homogeneous broadening of about $50 \mathrm{~nm}$ for the plasmon resonance at $710 \mathrm{~nm}$. The relationship between the plasmon resonance and its homogeneous broadening predict that the broadening is larger than $60 \mathrm{~nm}$ for a plasmon resonance at $800 \mathrm{~nm}$. Weaker nanosecond laser multi-pulse irradiation in Fales et al.'s experiment makes a spectral hole with a broadening of about $80 \mathrm{~nm}$ at $800 \mathrm{~nm}$ in the spectrum of gold nanorods ${ }^{48}$. Therefore, $80 \mathrm{~nm}$ was chosen as the reference value of spectral hole burning width at weak light limit.

\section{Discussion}

From our experiments, the damping coefficient can be estimated from the hole burning results: 

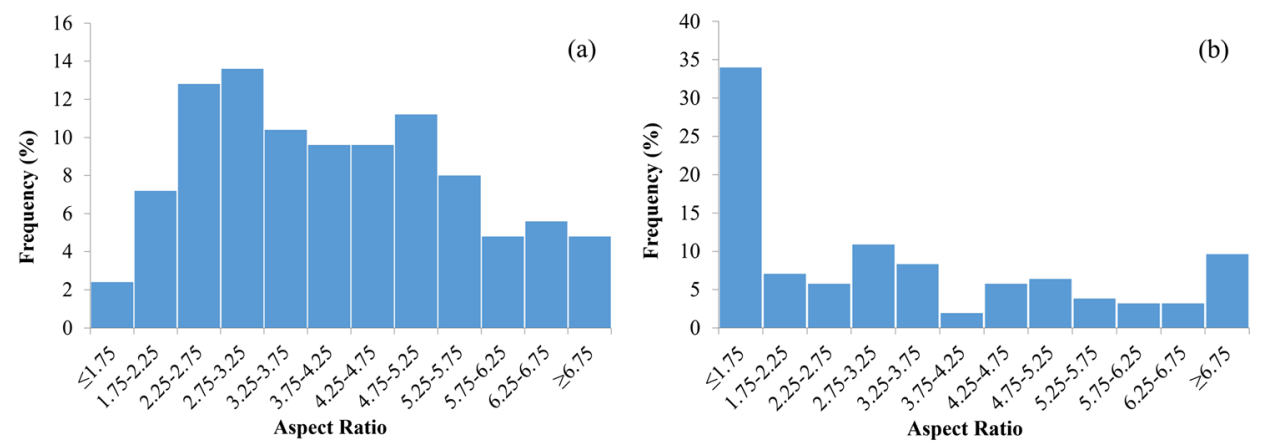

Figure 4. Histogram of aspect ratio before (a) and after (b) laser irradiation. Nanorods with aspect ratio near 4 reduces in portion, while significant amounts of nanorods with larger aspect ratio $(>6.75)$ remain.

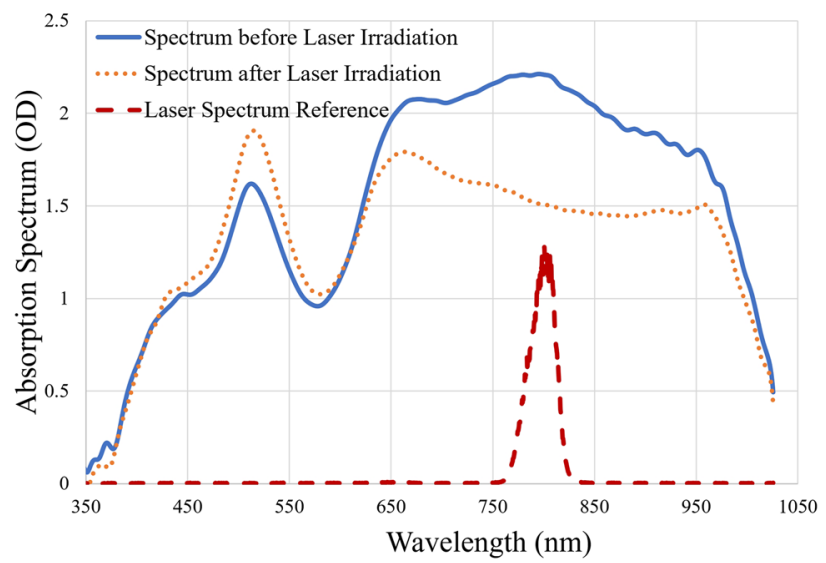

Figure 5. Absorption spectra of nanorod mixture before and after laser irradiation at fluence of $300 \mathrm{~J} / \mathrm{m}^{2}$.

$$
\mathrm{d} \omega=\frac{|\mathrm{d} \lambda| 2 \pi c}{\lambda^{2}}
$$

where $d \omega$ is the change in frequency, the damping coefficient is $\Gamma$ in this case, $\mathrm{d} \lambda$ is the spectral width from hole burning experiments, $\lambda$ is the wavelength of the spectral hole peak, and $c$ is the speed of light. The damping coefficients obtained at different laser fluences are presented in Fig. 8 with dots.

The interactions between a radiation electric field and free electrons in a metal is well described with the Drude model $^{49}$. The displacement $r$ of an electron in the electric field E follows Newton's second law,

$$
m_{e} \frac{d^{2} r}{d t^{2}}=-e E(t)-m_{e} \Gamma \frac{d r}{d t}
$$

where $m_{e}$ and $-e$ are the mass and electric charge of an electron, respectively, and $\Gamma$ is a damping coefficient that is decided by various scatterings of the electron in its motion. If a harmonic field $E=E_{0} \mathrm{e}^{-i \omega t}$ is applied to Eq. (3), we can obtain the dielectric constant $\varepsilon(\omega)$ of the metal at frequency $\omega^{49}$,

$$
\varepsilon(\omega)=1-\frac{N e^{2}}{m \varepsilon_{0}} \cdot \frac{1}{\omega^{2}+i \Gamma \omega}
$$

where $\varepsilon_{0}$ is the vacuum dielectric constant and $N$ is the electron density in the metal. The optical absorption spectra of a gold nanorod in a medium can be calculated with the dielectric constant $\varepsilon(\omega)$ of gold, the aspect ratio for the nanorod, and the dielectric constant of the medium ${ }^{50}$. Two resonant plasmons modes, i.e., transverse mode and longitudinal mode exist in the absorption spectrum. The longitudinal mode largely depends on the aspect ratio for the nanorod and the dielectric constant of the medium. The line spectral width of the plasmon resonant peaks is determined by $\Gamma$, which is originated from the electron scattering with nanorod's surface $\Gamma_{e-\text { surface }}$, the scattering with phonons $\Gamma_{e-p h o n o n}$, and the scattering of the electron with another electron $\Gamma_{e-e}$. We have observed that the $\Gamma$ increases when the single pulse fluence increases. $\Gamma_{e-\text { surface }}$ cannot explain the change of $\Gamma$ since this only relates to the shape and size of the nanorods. $\Gamma_{e-\text { phonon }}$ cannot explain the increase, since observed $\Gamma$ is less than 100 femtosecond, which is shorter than the time it takes for absorbed energy to be transferred to 

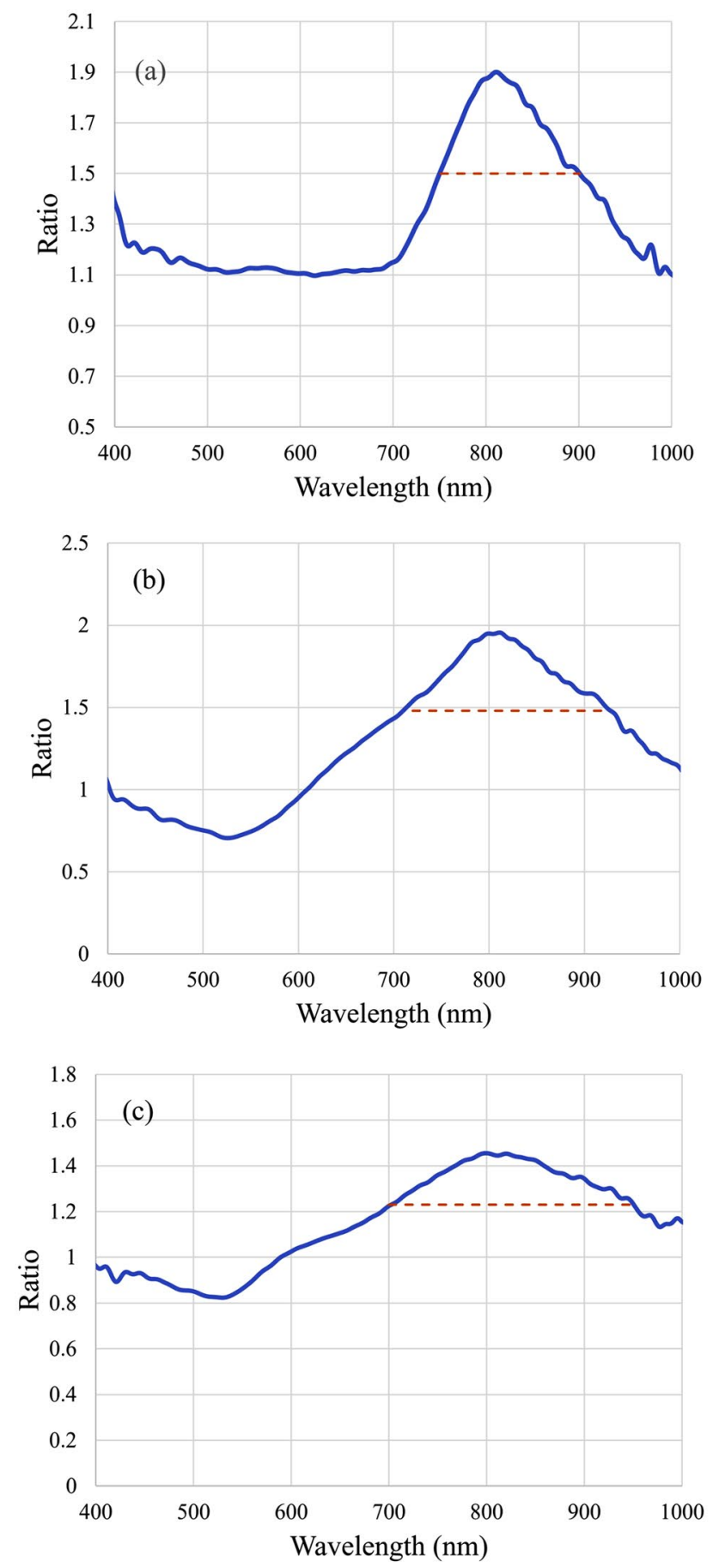

Figure 6. Ratio of absorption spectra before and after irradiating by laser with various fluences. (a) For $220 \mathrm{~J} /$ $\mathrm{m}^{2}$, (b) for $1.1 \times 10^{4} \mathrm{~J} / \mathrm{m}^{2}$, and (c) for $2.46 \times 10^{6} \mathrm{~J} / \mathrm{m}^{2}$. Widths of spectral holes are labeled with dashed lines.

the phonons from hot electrons ${ }^{37}$. For $\Gamma_{e-e}$, the electron-electron scattering rate was studied as early as in 1958, by Gurzhi ${ }^{51}$, and it was calculated in detail later in 1973 by Lawrence ${ }^{52}$. They used Born approximation and the Thomas-Fermi screening of Coulomb interaction to describe frequency and temperature dependence of the collision process among electrons ${ }^{51}$,

$$
\Gamma_{e-e}=\frac{\pi^{3}}{12 h E_{F}}\left[\left(k_{B} T\right)^{2}+\left(\frac{h \omega}{2 \pi}\right)^{2}\right]
$$

where $h$ is the Planck's constant, $k_{B}$ is the Boltzmann's constant, T is the temperature, and $E_{F}$ is the Fermi energy, for gold $E_{F}=5.5 \mathrm{eV}$. The calculation of the $T^{2}$ and $\omega^{2}$ dependences are based on the assumption that electrons 


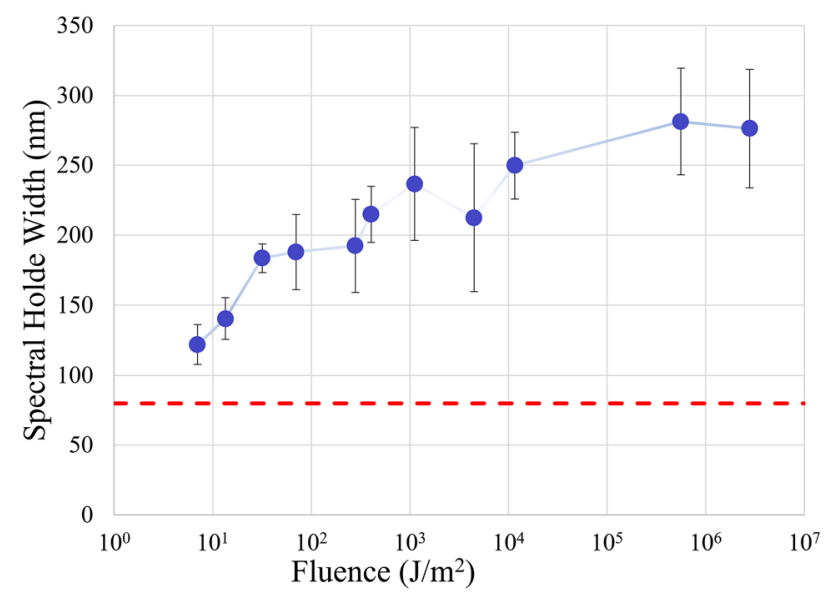

Figure 7. Laser fluence vs. hole burning spectral width in logarithm coordinate. Dots represent experimental data and the lines are simple connections between two nearest data points. Dashed line indicates natural resonance width at a low light intensity.

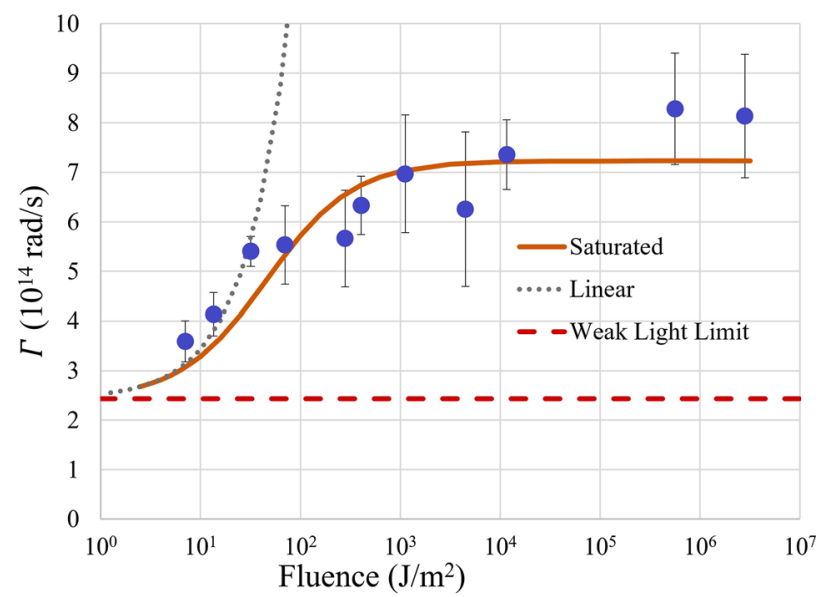

Figure 8. Laser fluence vs. damping coefficient $\Gamma$ in logarithm coordinate. Dots are experimental results; the solid line represents a fitting with a saturation function; the dotted line represents a fitting with a linear function at low fluence and the dashed line represents damping coefficient at weak light limit.

before and after scattering are close to the Fermi surface. By using the cross section of a gold nanorod ${ }^{41}$ and the laser fluence, we estimate that when the fluence is larger than $100 \mathrm{~J} / \mathrm{m}^{2}$, in the nanorod, the photon number is larger than the gold atoms. In such a high excitation where most of the electrons are excited, $E_{F}$ and $\mathrm{T}$ are not well defined since the system has not reached thermal equilibrium before scattering completes, and thus Eq. (5) cannot be used. At excitation, before the electron movement's dephasing, most of the electrons oscillate synchronously and the scattering event per electron among them should approach zero. Therefore, electron-electron scattering cannot explain the $\Gamma$ increase with fluence increase, either.

At such a high excitation, electrons are undergoing plasmonic oscillation together, and the photon number is so large that we cannot neglect the plasmon-photon interaction ${ }^{35}$. Plasmon and photon are both bosons, so when their numbers are large, the interaction becomes significant. Following Finazzi and Ciccacci's derivation ${ }^{35}$, with their the plasmon-photon interaction $H_{p l-p h}$, we consider the process: a plasmon in $\omega$ state with the occupation number of $n_{\omega}$ decaying into a photon in a state with wave vector $\boldsymbol{k}$ and polarization $\mathrm{u}_{\alpha}$ and occupation number of $\mathrm{n}_{\boldsymbol{k}, \alpha}$. The probability per unit time for a plasmon to decay into a photon with wave vector $\boldsymbol{k}$ and polarization $u_{\alpha}$ is given by the Fermi golden rule ${ }^{35}$ :

$$
\begin{aligned}
T_{\omega, \boldsymbol{k}, a} & =\frac{2 \pi}{\hbar}\left|n_{\omega}-1, n_{k, \alpha}+1\right| H_{p l-p h}\left|n_{\omega}, n_{k, \alpha}\right|^{2} \delta\left(\hbar \omega_{\omega}-\hbar \omega_{k}\right) \\
& =\frac{V_{p}}{2 \pi c^{3}} \omega_{k}^{4} n_{\omega}\left(n_{k, \alpha}+1\right)
\end{aligned}
$$


where $c$ is light speed, $\omega_{w}$ and $\omega_{\boldsymbol{k}}$ are the angular frequency of the plasmon and photon, and $V_{p}$ is the volume of a box containing the nanorod and the radiation. Because of this plasmon-photon interaction, the plasmon lifetime $\tau_{\mathrm{w}}$ is

$$
\tau_{w}=\frac{n_{w}}{T_{w, k, \alpha}}
$$

And the damping coefficient ${ }^{35}$ is

$$
\Gamma_{\omega}=\frac{1}{\tau_{\omega}}=\frac{V_{p} \omega_{k}^{4}}{2 \pi c^{3}}\left(n_{k, \alpha}+1\right)
$$

The factor $\left(n_{k, \alpha}+1\right)$ in Eq. (7) shows that $\Gamma$ increases as the light intensity increases. Subsequently, we introduce a simple linear relation where the $n_{k, \alpha}$ is proportional to the laser fluence $I$ :

$$
\Gamma=\Gamma_{0}+\alpha I
$$

where $\Gamma_{0}$ is damping coefficient when light intensity is very weak, $\alpha$ is a linear proportional coefficient. As shown in Fig. 8, in logarithm coordinate, the linear model is represented by an exponential curve, as shown by a dotted line. At a high intensity, the points deviate significantly from this trend since at a high fluence, this linear intensity dependence assumption from Eq. (8) fails and the rising trend slows. The photon occupation number $n_{k, \alpha}$ in Eq. (7) is not a simply linear function of laser fluence $I$. The photon occupation number $n_{k, \alpha}$ must be a monotonically increasing function of fluence $I$. To fit the experimental results, we may use:

$$
\Gamma=\Gamma_{0}+\frac{\alpha I}{1+\frac{I}{I_{s}}}
$$

where $I_{s}$ and $\alpha$ are the fitting parameters, $I_{s}$ is saturation intensity, and $\alpha$ is the same proportionality constant in Eq. (8), while $\Gamma_{0}$ is the low energy limit and is a constant that is $2.34 \times 10^{14} \mathrm{rad} / \mathrm{s}$. The fitted values are $I_{s}=46 \mathrm{~J} / \mathrm{m}^{2}$ and $\alpha=1.04 \times 10^{13} \mathrm{rad} \cdot \mathrm{m}^{2} \mathrm{~s}^{-1} \mathrm{~J}^{-1}$. The fitted curve is presented as the solid line. The trend matches the general behavior that $\Gamma$ is mostly linear at low fluences, exponential in logarithm coordinates, and then is saturated to a higher value. This saturation could be explained by simultaneous emission: that at very extreme intensity, plasmon interact with light and re-emit photons faster, and the duration of the pulse is longer than the lifetime of the plasmon, so exceeding energy would not contribute to photon occupation number fully.

\section{Conclusion}

A theory for plasmonic behavior based on the Drude model was built to relate spectral hole width with damping coefficient $\Gamma$. The persistent hole burning in the longitudinal mode band of gold nanorods irradiated by a single laser pulse shows that $\Gamma$ is tripled to about $7 \times 10^{14} \mathrm{rad} / \mathrm{s}$ at an average fluence of $10^{6} \mathrm{~J} / \mathrm{m}^{2}$, compared to $2 \times 10^{14} \mathrm{rad} / \mathrm{s}$ at weak light intensity. The results indicate that the damping coefficient increases sub-linearly with laser fluence. Using plasmon-photon interaction ${ }^{35}$, the increase of the damping coefficient was explained by the boson property of photons being ineligible when the photon number or light intensity is high. The experimental results show that the surface plasmonic effect holds at extreme femtosecond laser fluence, remaining $10 \%$ plasmonic enhancement of light at the fluence of about $10^{6} \mathrm{~J} / \mathrm{m}^{2}$.

Received: 1 September 2021; Accepted: 25 October 2021

Published online: 15 November 2021

\section{References}

1. Saha, K., Agasti, S., Kim, C., Li, X. \& Rotello, V. Gold nanoparticle-biological molecule interactions and catalysis. Chem. Rev. 112, 2739-2779 (2012).

2. Ghosh, P., Han, G., De, M., Kim, C. \& Rotello, V. Gold nanoparticles in delivery applications. Adv. Drug Deliv. Rev. (2008).

3. Austin, L. A., Kang, B. \& El-Sayed, M. A. Probing molecular cell event dynamics at the single-cell level with targeted plasmonic gold nanoparticles: A review. Nano Today 10(5), 542-558 (2015).

4. Murphy, C. et al. Gold nanoparticles in biology: Beyond toxicity to cellular imaging. Acc. Chem. Res. (2008).

5. Abadeer, N. S. \& Murphy, C. J. Recent progress in cancer thermal therapy using gold nanoparticles. J. Phys. Chem. C 120(9), 4691-4716 (2016).

6. Anker, J. N. et al. Biosensing with plasmonic nanosensors. In Nanoscience and Technology: A Collection of Reviews from Nature Journals 308-319 (World Scientific, 2010).

7. Jahn, M. et al. Plasmonic nanostructures for surface enhanced spectroscopic methods. Analyst 141(3), 756-793 (2016).

8. Wang, C. et al. Nature-like photosynthesis of water and carbon dioxide with femtosecond laser induced self-assembled metal nanostructures. Int. J. Mod. Phys. B 23(31), 5849-5857 (2009).

9. Wang, C., Shen, M., Huo, H., Ren, H. \& Johnson, M. Using metal nanostructures to form hydrocarbons from carbon dioxide, water and sunlight. AIP Adv. 1(4), 042124 (2011).

10. Chen, X.-J., Cabello, G., Wu, D.-Y. \& Tian, Z.-Q. Surface-enhanced Raman spectroscopy toward application in plasmonic photocatalysis on metal nanostructures. J. Photochem. Photobiol. C 21, 54-80 (2014).

11. Wang, C. et al. Low-cost visible-light photosynthesis of water and adsorbed carbon dioxide into long-chain hydrocarbons. Chem. Phys. Lett. 739, 136985 (2020).

12. Zhu, Q. et al. Conversion of water and carbon dioxide into methanol with solar energy on Au/Co nanostructured surfaces. Mater. Res. Express 7, 035014 (2020).

13. Barsotti, R. J. Jr. et al. Assembly of metal nanoparticles into nanogaps. Small 3(3), 488-499 (2007).

14. Cho, E. S. et al. Ultrasensitive detection of toxic cations through changes in the tunnelling current across films of striped nanoparticles. Nat. Mater. 11(11), 978-985 (2012).

15. Amendola, V. \& Meneghetti, M. Advances in self-healing optical materials. J. Mater. Chem. 22(47), 24501-24508 (2012). 
16. Luk'yanchuk, N. et al. The Fano resonance in plasmonic nanostructures and metamaterials. Nat. Mater. 9(9), 707-715 (2010).

17. Kraus, W. \& Schatz, G. C. Plasmon resonance broadening in small metal particles. J. Chem. Phys. 79(12), 6130-6139 (1983).

18. Apell, P. \& Penn, D. R. Optical properties of small metal spheres: Surface effects. Phys. Rev. Lett. 50(17), 1316 (1983).

19. Amendola, V., Pilot, R., Frasconi, M., Maragò, O. M. \& Iatì, M. A. Surface plasmon resonance in gold nanoparticles: A review. J. Phys. Condens. Matter 29(20), 203002 (2017).

20. Kennedy, B., Spaeth, S., Dickey, M. \& Carron, K. Determination of the distance dependence and experimental effects for modified SERS substrates based on self-assembled monolayers formed using alkanethiols. J. Phys. Chem. B 103(18), 3640-3646 (1999).

21. Gramotnev, D. K. \& Bozhevolnyi, S. I. Nanofocusing of electromagnetic radiation. Nat. Photon. 8(1), 13-22 (2014).

22. Hao, E. \& Schatz, G. C. Electromagnetic fields around silver nanoparticles and dimers. J. Chem. Phys. 120(1), 357-366 (2004).

23. Perner, M. et al. Homogeneous line widths of surface plasmons in gold nanoparticles measured by femtosecond pump-and-probe and near-field optical spectroscopy. J. Lumin. 76, 181-184 (1998).

24. Qiu, L. et al. Observation of plasmon line broadening in single gold nanorods. Appl. Phys. Lett. 93(15), 153106 (2008).

25. Shen, M., Oda, M. \& Goto, T. Direct evidence for photoionization in CdTe nanocrystals embedded in trioctylphosphine oxide. Phys. Rev. Lett. 82(19), 3915 (1999).

26. Horan, P. \& Blau, W. Photodarkening effect and the optical nonlinearity in a quantum-confined, semiconductor-doped glass. JOSA B 7(3), 304-308 (1990).

27. Empedocles, S. A. \& Bawendi, M. G. Quantum-confined stark effect in single CdSe nanocrystallite quantum dots. Science 278(5346), 2114-2117 (1997).

28. Shen, M., Goto, T., Kurtz, E., Zhu, Z. \& Yao, T. Photoluminescence properties of single CdSe quantum dots in ZnSe obtained by self-organized growth. J. Phys. Condens. Matter 10(10), L171 (1998).

29. Masumoto, Y. Persistent hole burning in semiconductor nanocrystals. J. Lumin. 70(1), 386-399 (1996).

30. Kudryashov, S., Samokhvalov, A., Shelygina, S. \& Veiko, V. Tuning of localized plasmon resonance in colloidal gold nano-particles by ultrafast interband photoinjection of free carriers: Superplasmonic states?. Appl. Phys. Lett. 115(16), 161903 (2019).

31. Link, S., Wang, Z. L. \& El-Sayed, M. A. How does a gold nanorod melt?. J. Phys. Chem. B 104(33), 7867-7870 (2000).

32. Desantis, C. J. et al. Laser-induced spectral hole-burning through a broadband distribution of Au nanorods. J. Phys. Chem. C 120(37), 150930151625000 (2015).

33. Link, S., Burda, C., Nikoobakht, B. \& El-Sayed, M. A. Laser-induced shape changes of colloidal gold nanorods using femtosecond and nanosecond laser pulses. J. Phys. Chem. B 104(26), 6152-6163 (2000).

34. Ditmire, T. et al. Nuclear fusion from explosions of femtosecond laser-heated deuterium clusters. Nature 398(6727), 489-492 (1999).

35. Finazzi, M. Plasmon-photon interaction in metal nanoparticles: Second-quantization perturbative approach. Phys. Rev. B Condens. Matter 86(3), 47-52 (2012).

36. Wang, X.-L. et al. Determination of air ionization threshold with femtosecond laser pulses. Acta Physica Sinica. 57(1), 354-357 (2008).

37. Kan, Z., Zhu, Q., Ren, H. \& Shen, M. Femtosecond laser-induced thermal transport in silicon with liquid cooling bath. Materials 12(13), 2043 (2019).

38. Shen, M., Crouch, C. H., Carey, J. E. \& Mazur, E. Femtosecond laser-induced formation of submicrometer spikes on silicon in water. Appl. Phys. Lett. 85(23), 5694-5696 (2004).

39. Zhu, Q. \& Shen, M. Femtosecond laser irradiation-induced infrared absorption on silicon surfaces. Int. J. Smart Nano Mater. 6(2), 113-123 (2015).

40. Loumaigne, M. et al. Optical extinction and scattering cross sections of plasmonic nanoparticle dimers in aqueous suspension. Nanoscale 8(12), 6555-6570 (2016).

41. Bohren, C. F. How can a particle absorb more than the light incident on it?. Am. J. Phys. 51(4), 323-327 (1983).

42. Welling, T. A. J. et al. Observation of undamped 3D Brownian motion of nanoparticles using liquid-cell scanning transmission electron microscopy. Part. Part. Syst. Charact. 2020, 2000003 (2020).

43. Rativa, D., De Araujo, R. \& Gomes, A. One photon nonresonant high-order nonlinear optical properties of silver nanoparticles in aqueous solution. Opt. Express 16(23), 19244-19252 (2008).

44. Klar, T. et al. Surface-plasmon resonances in single metallic nanoparticles. Phys. Rev. Lett. 80(19), 4249 (1998).

45. Johnson, P. B. \& Christy, R.-W. Optical constants of the noble metals. Phys. Rev. B 6(12), 4370 (1972).

46. Yeshchenko, O., Bondarchuk, I., Gurin, V., Dmitruk, I. \& Kotko, A. Temperature dependence of the surface plasmon resonance in gold nanoparticles. Surf. Sci. 608, 275-281 (2013).

47. Juvé, V. et al. Size-dependent surface plasmon resonance broadening in nonspherical nanoparticles: Single gold nanorods. Nano Lett. 13(5), 2234-2240 (2013).

48. Fales, A. M., Vogt, W. C., Wear, K. A., Ilev, I. K. \& Pfefer, T. J. Pulsed laser damage of gold nanorods in turbid media and its impact on multi-spectral photoacoustic imaging. Biomed. Opt. Express 10(4), 1919-1934 (2019).

49. Jackson, J. D. Classical Electrodynamics 3rd edn. (Wiley, 1999).

50. Link, S., Mohamed, M. \& El-Sayed, M. Simulation of the optical absorption spectra of gold nanorods as a function of their aspect ratio and the effect of the medium dielectric constant. J. Phys. Chem. B 103(16), 3073-3077 (1999).

51. Gurzhi, R., Azbel, M. Y. \& Lin, H. Surface effects in infrared optics. Sov. Phys. Solid State 5(3), 554-559 (1963).

52. Lawrence, W. Electron-electron scattering in the low-temperature resistivity of the noble metals. Phys. Rev. B 13(12), 5316 (1976).

\section{Acknowledgements}

This research was supported by the Army Research Office (W911NF1810172).

\section{Author contributions}

Z.W. and M.S. conceived the idea for the project. Z.W. performed the experiments. Z.W., Z.K. and M.S. discussed the results, analyzed the data, and drafted the manuscript. M.S. finalized the manuscript.

\section{Competing interests}

The authors declare no competing interests.

\section{Additional information}

Correspondence and requests for materials should be addressed to Z.W.

Reprints and permissions information is available at www.nature.com/reprints.

Publisher's note Springer Nature remains neutral with regard to jurisdictional claims in published maps and institutional affiliations. 
(c) (i) Open Access This article is licensed under a Creative Commons Attribution 4.0 International cc) License, which permits use, sharing, adaptation, distribution and reproduction in any medium or format, as long as you give appropriate credit to the original author(s) and the source, provide a link to the Creative Commons licence, and indicate if changes were made. The images or other third party material in this article are included in the article's Creative Commons licence, unless indicated otherwise in a credit line to the material. If material is not included in the article's Creative Commons licence and your intended use is not permitted by statutory regulation or exceeds the permitted use, you will need to obtain permission directly from the copyright holder. To view a copy of this licence, visit http://creativecommons.org/licenses/by/4.0/.

(C) The Author(s) 2021 